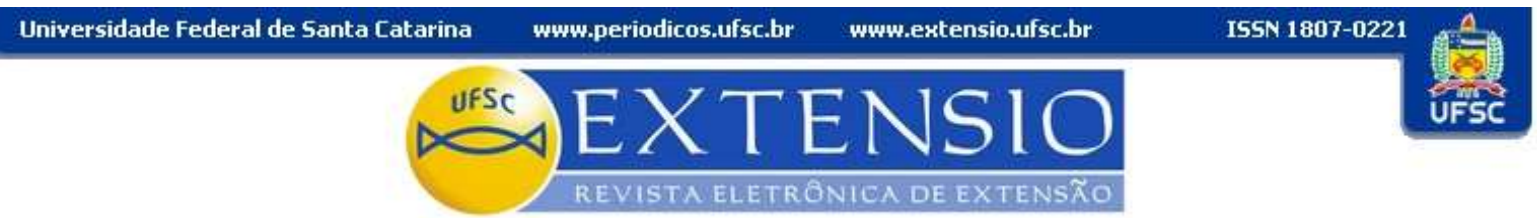

\title{
EDUCAÇÃO EM SAÚDE PARA GESTANTES E BEBÊS DE ALTO RISCO
}

\author{
Ana Marise Pacheco Andrade de Souza
}

Universidade Regional de Blumenau - FURB

amasouza@terra.com.br

\section{Rosana Silva dos Santos Schmitt}

Universidade Regional de Blumenau - FURB

volana@furb.br

Anamaria Araujo da Silva

Universidade Regional de Blumenau - FURB

anamariaas@terra.com.br

Anna Maria Dalmôninco Mose

Universidade Regional de Blumenau - FURB

annamdmoser@gamil.com

\section{Resumo}

Este trabalho é um relato de experiência de um programa de extensão intitulado Programa de Atenção à Saúde Materno-Infantil (PAMI), através de seu projeto "Educação em Saúde na Área Materno-Infantil", realizado junto às gestantes e bebês de alto risco atendidos no Ambulatório Universitário (AU) da Universidade Regional de Blumenau (FURB), localizada no Estado de Santa Catarina. O projeto atua desde 1996, e recebe apoio financeiro da PróReitoria de Pesquisa, Pós-Graduação, Extensão e Cultura (PROPEX). O objetivo é realizar a educação em saúde para gestantes de alto risco e mães de bebês de alto risco, oportunizando o conhecimento para a realização de ações e atitudes que promovam a qualidade de vida das mães e seus bebês. $\mathrm{O}$ trabalho tem caráter inter e multidisciplinar envolvendo profissionais e estudantes da odontologia, medicina, enfermagem, nutrição e psicologia. São realizados encontros na unidade de saúde, com temas selecionados a partir das necessidades elencadas pelas gestantes e mães dos bebês, além de atendimento psicológico conforme demanda e atendimento odontológico dos bebês. Esse trabalho propicia a troca de saberes entre todos os sujeitos que participam do processo e contribui para a melhoria da saúde física e mental das gestantes e dos bebês.

\section{(†) (ㄱ) Esta obra está licenciada sob uma Licença Creative Commons.}


Palavras-chave: Gestaçẩo de alto isco; Edectação em saúde; Bebês de alto risco; Interdisciplinaridade.

\title{
HEALTH EDUCATION FOR HIGH RISK PREGNANT WOMEN AND HIGH RISK INFANTS
}

\begin{abstract}
This paper is an experience report of in an extension program entitled Program for Attention to Maternal and Child Health (PAMI), through its project "Health Education in Maternal and Child Area", conducted among high risk pregnant women and high risk infants treated at the University Ambulatory (AU) of the University Regional de Blumenau (FURB), located in the state of Santa Catarina. The project has been active since 1996, and receives financial support from the Rectory of Research, Graduate Studies, Extension and Culture (PROPEX). The goal is to realize health education for high-risk pregnancies and mothers of high risk infants, creating opportunities for knowledge for performing actions and attitudes that promote quality of life for mothers and their babies. The work envolves an inter and multidisciplinary professionals and students of dentistry, medicine, nursing, nutrition and psychology. Meetings are held at the health unit, with selected topics from the needs listed by pregnant women and mothers of infants, as well as psychological demand and dental care of babies. This work promotes the knowledge exchange among all individuals that participate in the process and contribute to the improvement of physical and mental health of pregnant women and babies.
\end{abstract}

Keywords: High-risk pregnancy; Health education; Babies at high risk;Interdisciplinary. 
Educação em saúde para gestantes e bebês de alto risco

\section{INTRODUÇÃO}

A diversidade de contextos sócio-culturais e econômicos talvez se constitua no desafio maior para as universidades brasileiras. As mudanças que aconteceram no final do século passado levam as Universidades a repensarem o seu papel na sociedade, e buscar novos significados, pois num espaço privilegiado da geração do conhecimento técnico-científico, é intenção a formação de cidadãos capazes de responder às demandas da sociedade em geral, combinando-as com as de realização pessoal e não somente descrever ou explicar um estado de coisas (CARNEIRO, 2000).

A interdisciplinaridade nos Projetos de extensão também é uma nova forma de atuação, que segundo Vasconcelos (2001) passa a ser cobrada pela população e não apenas pelos profissionais. A articulação entre as ações interdisciplinares objetivam ampliar a visão deformada, parcial e limitada da realidade que o conhecimento em qualquer área, por maior que seja, representa (CARVALHO, 2003).

O conceito de extensão universitária, segundo a Política Nacional de Extensão Universitária (FORPROEX, 2012) é, sob o princípio constitucional da indissociabilidade entre ensino, pesquisa e extensão, "um processo interdisciplinar, educativo, cultural, científico e político que promove a interação transformadora entre Universidade e outros setores da sociedade".

A extensão pretende facilitar os momentos de ensino na sala de aula, assim como, os conteúdos do ensino servem de base para a pesquisa ou a extensão, afinal, é nesse subsídio teórico que a prática é embasada. A prática não pode ficar engessada na teoria, e a teoria por si só não se sustenta (GONZALES REY, 2002).

Programas nacionais voltados para a saúde da mulher e do bebê têm sido desenvolvidos, como a Rede Cegonha, que tem por objetivos: -fomentar a implementação de um novo modelo de atenção à saúde da mulher e saúde da criança com foco na atenção ao parto e ao nascimento e no desenvolvimento infantil de zero aos 24 meses; - organizar uma Rede de Atenção à Saúde Materna e Infantil que garanta acesso, acolhimento e resolutividade; e - reduzir a mortalidade materna e infantil, com ênfase no componente neonatal.

Dentro deste novo contexto a Universidade Regional de Blumenau (FURB) conta com um Projeto de Extensão de caráter educativo que atua de forma interdisciplinar, intitulado

\section{Esta obra está licenciada sob uma Licença Creative Commons.}


Educação em saúde para gestantes e bebês de alto risco

"Educação em Saúde na Área Materno-Infantil”, e é dirigido às gestantes, puérperas, acompanhantes e bebês atendidos no Ambulatório Universitário (AU) da FURB, e conta com a participação de professores e acadêmicos dos cursos de Psicologia, Odontologia, Nutrição e Medicina, além da enfermagem do AU.

Objetivando ações que promovam a saúde e qualidade de vida materno-infantil através do acompanhamento interdisciplinar do pré-natal e primeira infância, um dos focos do Projeto é o Aleitamento Materno. Os benefícios da amamentação com leite humano são bastante conhecidos e garantem, em muitos casos, a sobrevivência das crianças, em especial daquelas que vivem em condições desfavoráveis e/ou nasceram com baixo peso (MINISTÉRIO DA SAÚDE/ORGANIZAÇÃO PAN-AMERICANA DA SAÚDE, 2005).

A amamentação traz diversos benefícios para a mãe e para o bebê. A mulher que amamenta tem o retorno ao peso pré-gestacional mais rapidamente e o menor sangramento uterino pós-parto indicando menos chances de anemia (REA, 2004).

Segundo Ventura (2006) o profissional de saúde envolvido na assistência a mulheres e crianças tem como função promover o aleitamento materno na sua forma mais ampla, através da sensibilização, promoção, incentivo e apoio à prática. Ainda segundo o autor (2006) a gestação é a ocasião apropriada para promover a assistência integral à saúde da mulher e da criança.

Durante a gestação a mulher está mais susceptível a mudanças de hábitos, e sua conduta tem papel fundamental na educação da família (MAEDA et. al.., 2001; MOURA et. al.., 2001; SANTOS-PINTO et. al.., 2001). A atuação interdisciplinar neste período contribui para a preparação da futura mãe para uma gestação, parto e pós-parto de qualidade, considerando as especificidades culturais, psicossociais e econômicas que atravessam o fenômeno da maternidade.

Outro foco do projeto está na desvinculação do pré-natal visto somente como um ato médico, e sim ampliado para outras áreas de atenção à saúde.

A intervenção nutricional dada às gestantes do projeto por meio da educação nutricional justifica-se devido ao aumento da obesidade gerada pelo estilo de vida que os indivíduos vêm adquirindo, pelos hábitos alimentares errôneos, e pela falta de conhecimento do que seria uma alimentação saudável (SANTOS, 2005).

\section{Esta obra está licenciada sob uma Licença Creative Commons.}


Educação em saúde para gestantes e bebês de alto risco

O papel do nutricionista é, antes de tudo, o de educador. Educar, no âmbito da alimentação, implica em conhecer profundamente o que é alimentação, uma tarefa complexa, pois afeta atitudes e condutas. Atitudes são formadas por conhecimentos, crenças, valores e predisposições pessoais e sua modificação demanda reflexão, tempo e orientação competente (BOOG, 2005).

Atenção odontológica também tem sido dada às gestantes pelo fato da gravidez ser uma condição sistêmica que modifica o funcionamento do organismo como um todo e, em função disto, altera o equilíbrio bucal (OLIVEIRA; OLIVEIRA, 1999). A doença periodontal na gravidez tem recebido atenção especial pelo fato de predispor a gestante a um parto prematuro com bebês de baixo peso (SARTÓRIO; MACHADO, 2001).

É importante incentivar o atendimento odontológico durante a gestação assim como orientar a higiene bucal da gestante e educar para a futura higiene bucal dos bebês, a época da erupção dos dentes, seus sintomas, posição da amamentação, época do desmame.

O acompanhamento dos bebês na primeira infância permite a continuidade das ações iniciadas durante a gestação e a busca de um desenvolvimento integral destas crianças.

A Odontologia para Bebês visa a saúde bucal desde os primeiros meses de vida, adotando cuidados, para evitar o surgimento das doenças cárie (MARTINS et. al., 2001)e periodontal (CABRAL, 2001), e identificando problemas da oclusão ou outras alterações na boca do bebê (CAMARGO, 2001). O contato com o dentista deve começar com o aconselhamento e o exame clínico do bebê nos primeiros meses de vida, antes mesmo da erupção dos primeiros dentes decíduos.

O comparecimento precoce ao consultório odontológico possibilitará o trabalho conjunto dos pais e do odontopediatra na prevenção de futuros problemas dentários, e permitirá a orientação dos pais quanto aos cuidados com a limpeza da boca, o controle da amamentação noturna e do consumo de açúcar (RODRIGUES; CORRÊA; MATTOSGRANER, 2001).

O incentivo ao aleitamento materno inclui a promoção do correto desenvolvimento da musculatura orofacial e da dentição ((VENTURA, 2001), pois ao sugar o seio a criança estabelece o padrão correto de respiração nasal e deglutição. O uso de mamadeiras e chupetas poderão ocasionar alterações morfológicas e funcionais no desenvolvimento da criança.

\section{Esta obra está licenciada sob uma Licença Creative Commons.}


Educação em saúde para gestantes e bebês de alto risco

Depois de análise, e preocupada com os maiores problemas mundiais, a ONU propôs oito maneiras de mudar o mundo e as definiu em metas a serem atingidas até 2015, os chamados Objetivos do Milênio. Dentre eles destacam-se o $4^{\circ}$ (reduzir a mortalidade infantil) e o $5^{\circ}$ (melhorar a saúde das gestantes), objetivos que evidenciam a relevância do desenvolvimento do projeto.

Segundo a Política Nacional de Extensão Universitária (2012), além de todo o benefício gerado à comunidade, as atividades de Extensão Universitária contribuem decisivamente com a formação do estudante, seja ampliando o universo de referência que almejam, ou pelo contato direto com as grandes questões contemporâneas.

Este artigo relata a experiência de um programa de extensão intitulado Programa de Atenção à Saúde Materno-Infantil (PAMI), através de seu projeto "Educação em Saúde na Área Materno-Infantil", realizado junto às gestantes e bebês de alto risco atendidos no AU da FURB, localizada no Estado de Santa Catarina.

\section{MATERIAL E MÉTODOS}

$\mathrm{O}$ atendimento às gestantes é realizado uma vez por semana no AU da FURB, com gestantes de alto risco que realizam seu pré natal neste estabelecimento, que ao chegar serão encaminhadas para a verificação dos sinais vitais pela equipe de enfermagem do AU, e em seguida conduzidas à sala de reuniões onde acontece o grupo.

As gestantes participam de uma atividade grupal dialogada referente a temas relacionados à gestação e maternidade. Esta atividade ocorre sob a forma de conversas informais, tendo um caráter de troca de experiências, onde se procura relacionar o conhecimento popular com o científico. Os conteúdos emergentes no grupo e/ou temas geradores são abordados de forma multidisciplinar pelos membros da equipe, de acordo com a área envolvida. Nestes encontros podem participar companheiros e familiares das gestantes, incentivados pela equipe, principalmente em se tratando de gestantes adolescentes e de alto risco.

Orientações sobre higiene bucal e cuidados odontológicos durante a gestação são dadas por acadêmicos da Odontologia, além do encaminhamento a um serviço odontológico durante a gestação, se necessário, para a clínica de Odontologia Preventiva da FURB.

A equipe da psicologia está à disposição para atendimento individualizado conforme demanda. Como forma de intervenção nutricional para o tratamento e/ou prevenção de Esta obra está licenciada sob uma Licença Creative Commons. 
Educação em saúde para gestantes e bebês de alto risco

doenças e distúrbios nutricionais, são confeccionados materiais de orientação nutricional. É realizada a avaliação nutricional com as gestantes e após desenvolvido o processo educacional de conscientização e mudança de hábitos alimentares.

A grande dificuldade apresentada para o desenvolvimento das atividades das gestantes é a falta de um espaço específico, e, também, a dependência da data de agendamento estar sob dependência do funcionamento da disciplina de Ginecologia e Obstetrícia do Curso de Medicina.

As gestantes têm oportunidade de visitar o setor da Maternidade (centro obstétrico, maternidade e berçário) de uma unidade hospitalar de Blumenau com o objetivo de conhecer e minimizar dúvidas quanto ao processo do durante e pós-parto.

O grupo de puericultura ocorre semanalmente no AU, com os bebês (0 a 3 anos) da puericultura de risco. Com os bebês e seus pais são realizadas oficinas, como o álbum do bebê, além de entrevistas de acolhimento psicológico e prevenção em saúde bucal.

As ações objetivam a intervenção precoce na díade mãe-bebê. A partir das demandas e necessidades especificas de cada bebê, os mesmos são encaminhados para os demais serviços oferecidos pela equipe.

O principal problema enfrentado por nós é a falta de um espaço físico adequado para a realização de um questionário e orientações iniciais. Isto nos daria maior credibilidade e evitar-se-ia a dispersão das mães no momento das orientações.

O atendimento odontológico acontece na clínica de odontopediatria da FURB, onde as crianças serão atendidas individualmente com seus responsáveis e orientadas em relação aos hábitos bucais, higiene bucal,enfermidades bucais e examinadas.

Reuniões bimensais de avaliação da equipe são realizadas. A permanência de profissionais, professores e acadêmicos de várias áreas nos encontros são uma das características deste projeto.

\section{RESULTADOS E ANÁLISE}

O projeto de Educação em Saúde na Área Materno Infantil atua com gestantes e bebês de toda a região de Blumenau, pois o AU da FURB é o local de referência para a realização de pré-natal de alto risco, assim como da puericultura de alto risco.

\section{Esta obra está licenciada sob uma Licença Creative Commons.}


Educação em saúde para gestantes e bebês de alto risco

Cerca de quarenta gestantes e trinta e cinco bebês participam por semestre das atividades dos dois grupos.

O projeto serviu também para impulsionar o início de outro projeto, o de Formação Continuada em Aleitamento Materno, que trabalha em parceria com o Comitê Regional de Aleitamento Materno do Médio Vale do Itajaí, incentivando e promovendo o aleitamento materno através da formação de profissionais da rede básica de saúde da região. Através desta parceira foram realizados seis seminários regionais sobre o tema aleitamento.

A extensão resultou na integração com o ensino através do retorno dado em sala de aula pelos acadêmicos integrantes do projeto. Os acadêmicos também percebem um diferencial em sua formação pelo fato de estudarem e atenderem demandas que não surgem no dia a dia da graduação.

O projeto tem propiciado também o desenvolvimento de pesquisas de iniciação cientifica, mestrado e trabalhos de conclusão de curso. Foi finalizado recentemente um projeto de pesquisa referente ao Levantamento de Indicadores de Desmame Precoce em Bebês Atendidos pela Puericultura de Alto Risco do AU. O referido projeto resultou de uma necessidade de se investigar a questão do desmame precoce, que ocorre até o sexto mês de vida do bebê, cujos índices são altos nesta população, segundo o Ministério da Saúde. Como resultado da pesquisa percebeu-se que a hospitalização prolongada pareceu um indicador importante relacionado ao desmame precoce, uma vez que fragiliza o vínculo mãe-bebê, e consequentemente dificulta a prática da amamentação. Uma política relevante referente ao aleitamento materno se faz necessária, pois traz inúmeros benefícios para a díade mãe-bebê, e trabalha para diminuir os índices de desmame precoce.

Os resultados do projeto ainda podem ser medidos também pelo discurso das gestantes e mães que são atendidas e passam pelas atividades. Percebeu-se com o decorrer do tempo um aumento na procura pelas palestras que já começaram a fazer parte da rotina da instituição de saúde. Antes mesmo de serem informadas sobre a palestra as próprias gestantes já se perguntavam sobre o tema que seria abordado. A fala das gestantes durante a troca de experiências é indicador do interesse pelos temas abordados.

Apesar da falta de um espaço físico adequado dificultara execução das atividades, percebeu-se um esforço importante da gestão do AU no sentido de possibilitar que esse 
Educação em saúde para gestantes e bebês de alto risco

espaço fosse otimizado. Esse movimento vindo da própria instituição serve de indicador do impacto e relevância do projeto.

\section{CONSIDERAÇÕES FINAIS}

O presente projeto constitui uma possibilidade de investimento científico no campo da saúde materno-infantil, balizando suas ações, dentre outras, em prol de uma formação acadêmica mais ampliada, especificamente no que se refere ao redimensionamento de concepção e práticas em saúde coletiva.

A inserção de acadêmicos nas atividades da extensão implica em um diferencial na formação acadêmica, pois coloca o acadêmico em contato com a realidade e com as possibilidades de atuação em educação em saúde, exigindo uma capacidade crítica, compreensão da realidade, trabalho multidisciplinar, adaptabilidade a mudanças e criatividade.

O trabalho em equipe facilita à gestante superar essa fase de grandes alterações sofridas pela mulher além de conduzir e esclarecer dúvidas que surgem durante todo esse período. Assim como acompanhar a criança de risco ou não leva à prevenção do agravamento de situações que prejudicam o pleno desenvolvimento infantil.

Esta forma de trabalho redimensiona a abordagem grupal, pois o diálogo ocorre a partir de vários olhares práticos, técnicos e teóricos, sobre o mesmo fenômeno manifesto.

\section{REFERÊNCIAS}

BOOG, M. C. F. Os aspectos simbólicos da alimentação. Avisa lá, v. 5, p. 12-15, 2005.

CABRAL, F. C. In: CORRÊA, M. S. N. P. C. Odontopediatria na Primeira Infância. $2^{\text {a }}$ Reimpressão. São Paulo: Santos Livraria Editora, 2001.

CAMARGO, M. C. F. In: CORRÊA, M. S. N. P. C. Odontopediatria na Primeira Infância. $2^{a}$ Reimpressão. São Paulo: Santos Livraria Editora, 2001.

CARNEIRO, A. M. M. In: Metodologia e Experiência em projetos de Extensão, Niterói: EdUFF, 2000.

(7) (-) Esta obra está licenciada sob uma Licença Creative Commons. 
Educação em saúde para gestantes e bebês de alto risco

CARVALHO, G. D. S.O.S. Respirador Bucal: Uma visão funcional e clínica da amamentação. São Paulo: Editora Lovise, 2003.

FORPROEX - FÓRUM DE PRÓ-REITORES DE EXTENSÃO DAS UNIVERSIDADES PÚBLICAS BRASILEIRAS. POLÍTICA NACIONAL DE EXTENSÃO UNIVERSITÁRIA. Manaus-AM. Maio de 2012. Disponível em http://www.proec.ufpr.br/downloads/extensao/2012/legislacao/Politica\%20Nacional\%20de\% 20Extensao\%20Universitaria\%20maio2012.pdf Acesso em 16 de novembro de 2012.

GONZALES REY, F. L. Pesquisa qualitativa em psicologia: caminhos e desafios. Tradução: Marcel Aristides Ferrada Silva. São Paulo- Pioneira Thomson Learnig, 2002.

MAEDA, F. H. I.; TOLEDO, L. P.; PANDOLFI, M. A visão das gestantes quanto às condutas odontológicas na cidade de franca (SP). UFES - Revista de Odontologia Universidade Federal do Espírito Santo, v. 3, n. 2, p. 8-14, jul./dez. 2001.

MARTINS, A. L. C. F. et. al..In: CORRÊA, M. S. N. P. C. Odontopediatria na Primeira Infância. $2^{a}$ Reimpressão. São Paulo: Santos Livraria Editora, 2001.

MINISTÉRIO DA SAÚDE/ORGANIZAÇÃO PAN-AMERICANA DA SAÚDE. Guia alimentar para crianças menores de dois anos. Brasília: Ministério da Saúde/ Organização Pan-Americana da Saúde; 2005.

MOURA, L. F. A. D. et. al.. Apresentação do programa preventivo para gestantes e bebês. Jornal Brasileiro de Odontopediatria \& Odontologia do Bebê, v. 4, n. 17, p. 10-14, jan./fev. 2001.

OliveIRA, A. C. A. P.; OLIVEIRA, A. F. B. Saúde bucal em gestantes: um enfoque educativo - preventivo. Jornal Brasileiro de Odontopediatria \& Odontologia do Bebê, v. 2, n. 7, p. 182-185, mai./jun. 1999.

ORGANIZAÇÃO DAS NAÇÕES UNIDAS - ONU. Objetivos do milênio. Disponível em: http://www.objetivosdomilenio.org.br/ ODM. Acesso em 12 de novembro de 2012.

REA M. F. Os benefícios da amamentação para a saúde da mulher. Jornal de Pediatria,Rio de Janeiro, v. 80, n. 5, p. 142-146, 2004.

\section{c) (i) \\ Esta obra está licenciada sob uma Licença Creative Commons.}


Educação em saúde para gestantes e bebês de alto risco

REDE

CEGONHA.

Disponível

em:

http://portal.saude.gov.br/portal/saude/Gestor/visualizar_texto.cfm?idtxt=37472\&janela=1

Acesso em 13 de novembro de 2012.

RODRIGUES, C. R. M. D.; CORRÊA, M. S. N. P.; MATTOS-GRANER, R. O. In: CORRÊA, M. S. N. P. C. Odontopediatria na Primeira Infância. $2^{\text {a }}$ Reimpressão. São Paulo: Santos Livraria Editora, 2001.

SANTOS L.A.S. Educação alimentar e nutricional no contexto da promoção de práticas alimentares saudáveis. Rev. Nutr., Campinas, v.18, n.5, p. 681-692, set./out., 2005.

SANTOS-PINTO, L. et. al.. O que as gestantes conhecem sobre a saúde bucal? Jornal Brasileiro de Odontopediatria \& Odontologia do Bebê, v. 4, n. 21, p. 427-434, set./out. 2001.

SARTORIO, M. L.; MACHADO, W. A. S. Doença periodontal na gravidez. Revista Brasileira de Odontologia, v. 58, n. 5, p. 306-308, set./out. 2001.

VASCONCELOS, E. A saúde nas palavras e nos gestos: reflexões da rede de educação popular e saúde. São Paulo: Editora Hucitec, 2001.

VENTURA, W. P. Promovendo o Aleitamento Materno no Pré-natal, Pré-parto e Nascimento. In: REGO, J.D. Aleitamento Materno. 2a ed. São Paulo: Editora Atheneu, 2006. 